\title{
On D. G. Higman's note on regular 3-graphs
}

\author{
Daniel Kalmanovich \\ Department of Mathematics \\ Ben-Gurion University of the Negev \\ 84105 Beer Sheva, Israel
}

Received 17 October 2011, accepted 25 April 2012, published online 4 June 2012

\begin{abstract}
We introduce the notion of a $t$-graph and prove that regular 3-graphs are equivalent to cyclic antipodal 3 -fold covers of a complete graph. This generalizes the equivalence of regular two-graphs and Taylor graphs. As a consequence, an equivalence between cyclic antipodal distance regular graphs of diameter 3 and certain rank 6 commutative association schemes is proved. New examples of regular 3-graphs are presented.
\end{abstract}

Keywords: Antipodal graph, association scheme, distance regular graph of diameter 3, GodsilHensel matrix, group ring, Taylor graph, two-graph.

Math. Subj. Class.: 05E30, 05B20, 05E18

\section{Introduction}

This paper is mainly a clarification of [6] - a short draft written by Donald Higman in 1994, entitled "A note on regular 3-graphs".

The considered generalization of two-graphs was introduced by D. G. Higman in [5]. As in the famous correspondence between two-graphs and switching classes of simple graphs, $t$-graphs are interpreted as equivalence classes of an appropriate switching relation defined on weights, which play the role of simple graphs.

In his note Higman uses certain association schemes to characterize regular 3-graphs and to obtain feasibility conditions for their parameters. Specifically, he provides a graph theoretic interpretation of a weight and from the resulted graph he constructs a rank 4 symmetric association scheme and a rank 6 fission of it. Furthermore, he proves that rank 6 schemes with parameters as in his construction are equivalent to regular 3-graphs.

During our redetermination of the structure constants of the rank 6 scheme an error in [6] was detected, this miscalculation led Higman to a false restriction on the parameters

E-mail address: dannykal@bgu.ac.il (Daniel Kalmanovich) 
of regular 3-graphs. Our first contribution is the correction of this mistake (see Subsection 5.2). The second contribution is a proof (see Section 4) that in the case of regular 3 -graphs, the graph defined by a weight in its switching class is a distance regular cover of the complete graph. Moreover, it is a cyclic antipodal distance regular (ADRG) 3-fold cover of the complete graph in the sense of Godsil and Hensel in [2]. This provides a further restriction on the parameters of regular 3-graphs.

Altogether, in Section 4 and in Section 5 we establish a one-to-one correspondence between regular 3 -graphs, cyclic ADRGs of diameter 3 and certain rank 6 association schemes. As a consequence, we provide a new characterization of cyclic antipodal distance regular 3 -fold covers of the complete graph in terms of association schemes.

To keep the length of this paper reasonable we did not include all necessary preliminaries. In particular, we assume some knowledge of distance regular graphs, specifically, antipodal distance regular graphs of diameter 3. Also, we assume the reader is familiar with association schemes, in particular, the intersection algebra of an association scheme and its character-multiplicity table. An interested reader may find a more comprehensive consideration of all the diverse links exposed below as well as suggestions for further research in [7].

\section{Two-graphs and $t$-graphs}

\subsection{Two-graphs and regular two-graphs}

Two-graphs have roots originating in diverse areas of combinatorics, geometry and group theory, thus leading to different manifestations in the literature, such as: switching classes of graphs, sets of equidistant points in elliptic geometry, sets of equiangular lines in Euclidean geometry, binary maps of triples with vanishing coboundary, and double coverings of complete graphs (see the celebrated survey [12]). Our focus will be on the last two interpretations and the connection between them. We start with the classical definition and the classical viewpoint of two-graphs as switching classes of simple graphs.

Let $X$ be a set of $n$ elements called vertices. For $m \in \mathbb{N}$ denote by $X^{\{m\}}$ the set of all $m$-subsets of $X$.

Definition 2.1. A set $\Delta \subseteq X^{\{3\}}$ is a two-graph if every 4-subset of $X$ contains an even $(\in\{0,2,4\})$ number of members of $\Delta$.

Typically we use the notation $(X, \Delta)$ for a two-graph, and call $\Delta$ the set of odd triples.

Definition 2.2. A two-graph $(X, \Delta)$ is called regular if every 2-subset $\{x, y\} \in X^{\{2\}}$ is contained in the same number of triples from $\Delta$.

The most famous view of two-graphs is related to a special equivalence relation that is defined on the set of simple (undirected, no loops) graphs. First we remind the reader how to get a two-graph from a graph:

Let $\Gamma=(V, E)$ be a simple graph. The set of triples $\{u, v, w\}$ of vertices, such that the induced subgraph $\left.\Gamma\right|_{\{u, v, w\}}$ has an odd number of edges, forms a two-graph.

Next, to define the equivalence relation we consider the operation of switching a graph with respect to a set of vertices.

Definition 2.3. Let $X \subseteq V$ be a subset of vertices of a simple graph $\Gamma=(V, E)$. Switching with respect to $X$ means interchanging the adjacencies and non-adjacencies between $X$ and its complement $V \backslash X$. 
As a more appropriate setting to work with the operation of switching, J. J. Seidel proposed an alternative matrix representation of a simple graph:

Definition 2.4. The Seidel adjacency matrix $S=\left(s_{i, j}\right)$ of a graph $\Gamma=(V, E)$ is a $\{0,-1,1\}$-matrix having:

$$
s_{i, j}=\left\{\begin{array}{cc}
0 & i=j \\
-1 & \{i, j\} \in E \\
1 & \{i, j\} \notin E
\end{array}\right.
$$

In this notation, if the graph $\Gamma^{\prime}$ is obtained from $\Gamma$ by switching with respect to $X \subseteq V$, then its Seidel adjacency matrix $S^{\prime}$ is obtained from $S$ via a similarity transformation by a diagonal matrix having $\{-1,1\}$ on its diagonal. Explicitly:

$$
S^{\prime}=D S D
$$

where $D_{i, i}=-1 \Longleftrightarrow i \in X$.

As was implied above, switching is an equivalence relation on the set of all simple graphs of order $n$ sharing the same prescribed vertex set. Furthermore we note that switching equivalent graphs give rise to the same two-graph, and have the same Seidel spectrum, thus allowing us to define the eigenvalues and their multiplicities of a two-graph. To sum up we have:

Theorem 2.5. There is a 1-1 correspondence between two-graphs and switching classes of graphs.

Theorem 2.6. A two-graph is regular if and only if it has two distinct (Seidel) eigenvalues $\rho_{1}>0>\rho_{2}$, such that $\rho_{1} \rho_{2}=1-|X|$.

The following is an alternative definition of a two-graph. We call it the cohomological definition for reasons that will be clear soon.

Definition 2.7. Let $U_{2}$ be the group of square roots of unity. A set $\Delta \subseteq X^{\{3\}}$ is a twograph if the function:

$$
f: X^{\{3\}} \longrightarrow U_{2}
$$

defined by

$$
f(x)=-1 \Longleftrightarrow x \in \Delta,
$$

satisfies:

$$
f(\{x, y, z\}) \cdot f(\{x, y, t\}) \cdot f(\{x, z, t\}) \cdot f(\{y, z, t\})=1
$$

for any $\{x, y, z, t\} \in X^{\{4\}}$.

Functions satisfying the equation in the above definition are called 3-cocycles (see below).

It is clear that the two definitions are equivalent. Furthermore, we may refer to either $(X, \Delta), \Delta$ or the function $f$ as the two-graph. 


\subsection{The connection with double covers of complete graphs}

Two-graphs were originally introduced by Graham Higman to study 2-transitive representations of certain sporadic groups, in his description he used antipodal 2-fold covers of complete graphs. In [16], Taylor and Levingston established a one-to-one correspondence between two-graphs and antipodal 2-fold covers of complete graphs. This correspondence will be described in a more general setting with all details in the next section. Meanwhile we give an overview for the case of two-graphs.

Let $\Gamma$ be a graph with $n$ vertices in the switching class of the two-graph $f$ and let $S_{\Gamma}$ be the Seidel adjacency matrix of $\Gamma$. Then by inserting a $2 \times 2$ matrix in the place of each entry of $S_{\Gamma}$ according to the following rule:

$$
0 \longleftrightarrow\left(\begin{array}{ll}
0 & 0 \\
0 & 0
\end{array}\right) \quad 1 \longleftrightarrow\left(\begin{array}{ll}
1 & 0 \\
0 & 1
\end{array}\right) \quad-1 \longleftrightarrow\left(\begin{array}{ll}
0 & 1 \\
1 & 0
\end{array}\right)
$$

we obtain a $2 n \times 2 n\{0,1\}$-matrix which is the usual adjacency matrix of the corresponding 2-fold cover of $K_{n}$. The converse construction is done in a similar manner: substituting each $2 \times 2$ block of the adjacency matrix of a 2 -fold cover of $K_{n}$ (writing it in a suitable ordering of the vertices) with an element of $\{0,1,-1\}$.

A 2 -fold cover of $K_{n}$, when it is also distance regular, is called a Taylor graph, these are distance regular graphs with intersection array

$$
\{k, \mu, 1 ; 1, \mu, k\}
$$

In the above mentioned correspondence, Taylor graphs correspond to regular twographs. This will be a particular case of our more general result later on.

\subsection{Generalizing two-graphs}

Considering the cohomological definition of two-graphs, two very natural generalizations arise:

- $t$-cocycles into $U_{2}$ (functions $f: X^{\{t\}} \longrightarrow U_{2}$ with a similar property as for two-graphs);

- 3-cocycles into $U_{t}$ (functions $f: X^{\{3\}} \longrightarrow U_{t}$, where $U_{t}$ is the group of $t$-th roots of unity).

Historically, the first of these was indeed the first to be considered. The first appearance of the term $t$-graph as a $t$-cocycle over $U_{2}$ is due to D. Higman's generalization (see [4]) of E. Shult's graph extension theorem (see [13]). Other sources of this (design theoretical) generalization can be found in Mielants [11] or in [1]. In this case, a regular $t$-graph is a $t$-cocycle into $U_{2}$ which is also a $t$-design. Here just few examples are known: regular 3 -graphs on 8 and 12 points and a regular 5-graph on 12 points (see [10]). Our interest in the current presentation is the second way to generalize two-graphs, i.e. 3-cocycles into $U_{t}$. This direction was examined by D. Higman, and the main source of this is [5]. We begin with introducing some very basic elements of cohomology theory, in which terms $t$-graphs are defined. 


\subsection{Some cohomology}

Let $X$ be a finite set with $|X|=n$. Let $\zeta$ be a primitive root of unity of order $t$, and let $U_{t}=\left\langle\zeta>\right.$ denote the cyclic group of $t^{t h}$ roots of unity generated by $\zeta$.

Let $x=\left(x_{1}, x_{2}, \ldots, x_{p}\right) \in X^{p}$. A function $f: X^{p} \longrightarrow U_{t}$ is called a $p$-cochain if:

(i) $f(x)=1$ (the identity element of $U_{t}$ ) for all $x \in X^{p}$ such that $x_{i}=x_{j}$ for some $1 \leq i \neq j \leq p$

(ii) if $y$ results from $x$ by interchanging $x_{i}$ and $x_{j}$ for some $1 \leq i \neq j \leq p$ then $f(y)=$ $(f(x))^{-1}$.

The set of all p-cochains together with pointwise multiplication forms a group denoted by $C$. $\left(X, U_{t}\right)$. Define the coboundary operator:

$$
\delta: C^{p}\left(X, U_{t}\right) \longrightarrow C^{p+1}\left(X, U_{t}\right)
$$

by

$$
\delta f(x)=\prod_{i=0}^{p} \sigma^{i}\left(f\left(\widehat{x}_{i}\right)\right)
$$

where $\widehat{x_{i}} \in X^{p}$ is obtained from $x \in X^{p+1}$ by deleting the $i^{t h}$ coordinate $x_{i}$, and $\sigma$ is the inverse operation of $U_{t}$.

For $e \in X$ and $p \geq 1$ we have the group homomorphism

$$
\Delta_{e}: C^{p}\left(X, U_{t}\right) \longrightarrow C^{p-1}\left(X, U_{t}\right)
$$

defined by

$$
\Delta_{e} f(x)=f(e, x)
$$

for $x \in X^{p-1}$.

Define the set of $p$-coboundaries:

$$
B^{p}\left(X, U_{t}\right)=\left\{\delta f \mid f \in C^{p-1}\left(X, U_{t}\right)\right\},
$$

and the set of $p$-cocycles:

$$
Z^{p}\left(X, U_{t}\right)=\left\{f \in C^{p}\left(X, U_{t}\right) \mid \delta f=\mathbb{1}\right\} .
$$

Here $\mathbb{1}$ is the identity cochain in $C^{p+1}\left(X, U_{t}\right)$. It is routine to check that $\delta^{2} f=\mathbb{1}$ for any $(p-1)$-cochain $f$, and thus the coboundary of any $(p-1)$-cochain is a $p$-cocycle. Two $(p-1)$-cochains have the same $p$-cocycle as their coboundary if and only if their quotient is a $(p-1)$-cocycle. Thus, $p$-cocycles correspond to cohomology classes of $(p-1)$-cochains, as a generalization of Seidel switching we call the cohomology classes switching classes.

Along the considered generalization of two-graphs and regular two-graphs we define:

Definition 2.8. A $t$-graph is a 3 -cocycle into $U_{t}$.

Definition 2.9. A $t$-graph is called regular if for every pair $x, y \in X$, the number of $z \in X \backslash\{x, y\}$ such that $f(x, y, z)=\alpha$ depends only on $\alpha \in U_{t}$. This number is denoted $m(\alpha)$.

It is easy to check that in case $t=2$, the definition of a 3-cocycle into $U_{2}$ is compatible with the characterization given in Definition 2.7, and that the above definition of regularity is compatible with Definition 2.2. 


\subsection{Weights}

According to Higman, a weight on $X$ with values in $U_{t}$ is a 2 -cochain $w \in C^{2}\left(X, U_{t}\right)$, from this point onward we will call them simply weights. Thus $t$-graphs are the coboundaries of weights. A weight $w$ can be represented as a $n \times n$ matrix $W$ with entries from $U_{t}$ where:

$$
(W)_{x, y}=w(x, y)
$$

Then $W$ has 1 on its diagonal, and $W^{*}=W$, where $W^{*}$ is obtained from $W$ by transposing and inverting each entry. We will investigate the matrix representation of a weight with much more detail in the next section where we will focus on the case $t=3$.

Another way to represent a weight is as an antipodal $t$-fold cover of $K_{n}$.

Definition 2.10. Let $w: X^{2} \longrightarrow U_{t}$ be a weight on $X$. To each element $x \in X$ we associate $t$ vertices $x_{1}, x_{2}, \ldots, x_{t}$ and define a graph $\Gamma_{w}=(V, E)$ on the resulting set $V$ of $t|X|$ vertices by

$$
\left\{x_{i}, y_{j}\right\} \in E \Longleftrightarrow w(x, y)=\zeta^{j-i} \text {. }
$$

The resulting graph is a $t$-fold cover of the complete graph $K_{n}$, and if $w(x, y)=\zeta^{i}$ then the set of edges between $x_{1}, x_{2}, \ldots, x_{t}$ and $y_{1}, y_{2}, \ldots, y_{t}$ forms a perfect matching which is given by the $i^{t h}$ power of the permutation matrix of $(1,2, \ldots, t)$. Permuting $x_{1}, x_{2}, \ldots, x_{t}$ according to some power of the permutation $(1,2, \ldots, t)$ amounts to a change of $w$ in its switching class.

\section{Regular 3-graphs}

From now on we focus on the case $t=3$. We will prove that the situation for regular 3 -graphs generalizes the case of regular two-graphs. In particular, regular 3-graphs are in $1-1$ correspondence with regular (cyclic) $\left(n, 3, c_{2}\right)$-covers.

\subsection{Main conventions}

Let $w: X^{2} \longrightarrow U_{3}$ be a weight on $X$ and $|X|=n$. The coboundary $\delta w$ of $w$ is a 3-graph $\Phi \in Z^{3}\left(X, U_{3}\right)$ on $X$. Assume that $\Phi$ is regular. Recall that this means that for every pair $x, y$ of distinct elements of $X$ and $\alpha \in U_{3}$, the number $m(\alpha)$ of $z \in X \backslash\{x, y\}$ such that $\Phi(x, y, z)=\alpha$ is independent of the choice of $x$ and $y$.

Denote

$$
\begin{aligned}
& a:=m(\mathbf{1}), \\
& b:=m(\zeta)=m\left(\zeta^{2}\right) .
\end{aligned}
$$

We call $(n, a, b)$ the parameters of the regular 3-graph $\Phi$. We obtain the first restriction on the parameters by simple counting. Fix two vertices $x, y \in X$, then:

$$
\begin{aligned}
|X \backslash\{x, y\}| & =|\{z \in X \mid \Phi(x, y, z)=1\}| \\
& +|\{z \in X \mid \Phi(x, y, z)=\zeta\}|+\left|\left\{z \in X \mid \Phi(x, y, z)=\zeta^{2}\right\}\right| .
\end{aligned}
$$

Thus we have

$$
n-2=a+2 b
$$


The corresponding graph $\Gamma_{w}$ is a 3 -fold cover of $K_{n}$ with exactly 3 types of matchings between fibres:

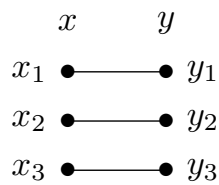

$w(x, y)=\mathbf{1}$

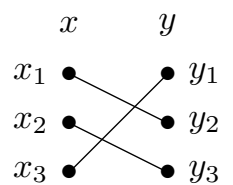

$w(x, y)=\zeta$

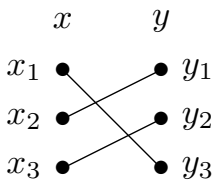

$w(x, y)=\zeta^{2}$

Figure 1: Matchings between fibres of $\Gamma_{w}$

The following subsection serves to remind and fix notation about matrices over the integral group ring, which is the setting in which we characterize regular 3-graphs.

\subsection{Matrices over group rings}

Let $T$ be a finite group. The elements of the integral group ring $\mathbb{Z}[T]$ are expressions of the form

$$
\sum_{g \in T} a_{g} g
$$

where $a_{g} \in \mathbb{Z}$. The ring operations are:

$$
\begin{gathered}
\left(\sum_{g \in T} a_{g} g\right)+\left(\sum_{g \in T} b_{g} g\right)=\sum_{g \in T}\left(a_{g}+b_{g}\right) g, \\
\left(\sum_{g \in T} a_{g} g\right) \cdot\left(\sum_{h \in T} b_{h} h\right)=\sum_{g, h \in T}\left(a_{g} \cdot b_{h}\right) g h .
\end{gathered}
$$

Following the notation of Klin and Pech in [8], for a subset $M \subseteq T$ define the simple quantity $\underline{M} \in \mathbb{Z}[T]$ :

$$
\underline{M}=\sum_{m \in M} 1 \cdot m \text {. }
$$

When $M=\{g\}$ we will slightly abuse notation and write $g$ instead of $\{g\}$. The multiplicative identity of $\mathbb{Z}[T]$ is $\underline{\mathbf{1}}$ where $\mathbf{1}$ is the identity of $T$. The adjoint of $\sum_{g \in T} a_{g} g$ is

$$
\left(\sum_{g \in T} a_{g} g\right)^{*}=\sum_{g \in T} a_{g} g^{-1}
$$

The set of $n \times n$ matrices with entries from $\mathbb{Z}[T]$ is denoted by $\mathbb{Z}[T]^{n \times n}$. This set together with usual addition and multiplication of matrices forms a ring with identity. Moreover, $\mathbb{Z}[T]^{n \times n}$ forms a $\mathbb{Z}[T]$-module, and for a matrix $A=\left(a_{i, j}\right) \in \mathbb{Z}[T]^{n \times n}$ we can define the adjoint $A^{*} \in \mathbb{Z}[T]^{n \times n}$, where

$$
\left(a_{i, j}\right)^{*}=a_{j, i}^{*}
$$


Recall, that a matrix $A$ is called self-adjoint if $A=A^{*}$.

\subsection{Godsil-Hensel matrices}

Let $\Gamma$ be a connected cover of some graph $\Delta$ and consider the group $T$ of all automorphisms of $\Gamma$ that fix each fibre of $\Gamma$ setwise. Then $T$ acts semi-regularly on $V(\Gamma)$ (cf. [2, Sec. 7]), and in particular on each fibre of $\Gamma$. The group $T$ is called the voltage group of $\Gamma$. If $T$ acts regularly on each fibre, then $\Gamma$ is called a regular cover of $\Delta$.

In [2] Godsil and Hensel studied regular covers in general and in particular gave a characterization of regular antipodal distance regular covers of complete graphs. For this purpose they defined certain matrices over the integral group ring $\mathbb{Z}[T]$, that we will introduce below in the notation used by Klin and Pech in [8].

Let $A=\left(a_{i, j}\right) \in \mathbb{Z}[T]^{n \times n}$ be a matrix such that $a_{i, j} \in(\{g \mid g \in T\} \cup\{0\})$, all elements on the diagonal are equal to 0 , and such that $A$ is self-adjoint. Then to $A$ we can associate two graphs:

1) the underlying graph $\Delta_{A}$ with vertex set $V\left(\Delta_{A}\right)=\{1, \ldots, n\}$ and edge set $E\left(\Delta_{A}\right)=$ $\left\{\{i, j\} \mid a_{i, j} \neq 0\right\}$

2) the derived graph $\Gamma^{A}$ with vertex set $V\left(\Gamma^{A}\right)=\{1,2, \ldots, n\} \times T$ and edge set $E\left(\Gamma^{A}\right)=$ $\left\{\{(i, g),(j, h)\} \mid a_{i, j} \neq 0\right.$, and $\left.\underline{g} \cdot a_{i, j}=\underline{h}\right\}$.

Such matrices, when defining connected covers with voltage group $T$, are called covering matrices. When $\Delta_{A}$ is a complete graph $K_{n}$, and $\Gamma^{A}$ is an $\left(n, r, c_{2}\right)$-cover of $\Delta_{A}$ then the matrix $A$ is called the Godsil-Hensel matrix of the cover.

Theorem 3.1. Let $T$ be a finite group and let $A$ be a covering matrix of order $n$ over $T$. Then $A$ is the Godsil-Hensel matrix of a regular antipodal $\left(n, r, c_{2}\right)$-cover of $K_{n}$ with voltage group $T$ if and only if

$$
A^{2}=(n-1) I+\left(n-2-r c_{2}\right) A+c_{2} \underline{T}(J-I) .
$$

\section{Main results}

Throughout this section we let $\Phi$ denote a regular 3-graph, $w$ a weight such that $\delta w=$ $\Phi$. Let $\Gamma_{w}$ be the antipodal 3 -fold cover of $K_{n}$ defined by $w$ and let $W$ be the matrix representation of $w$.

Lemma 4.1. Let $\Phi$ be a regular 3-graph and let $w$ be a weight with $\delta w=\Phi$. Then $W$ satisfies:

$$
\begin{aligned}
W^{2} & =n I+\left((a+2) \underline{\mathbf{1}}+b \underline{\zeta, \zeta^{2}}\right)(W-I) \\
& =n I+(a+2-b) \underline{\mathbf{1}}(W-I)+b \underline{U_{3}}(J-I) .
\end{aligned}
$$


Proof. We calculate $\left(W^{2}\right)_{x, y}$. For $x=y$ we have:

$$
\begin{aligned}
\left(W^{2}\right)_{x, x} & =\sum_{z \in X}(W)_{x, z} \cdot(W)_{z, x} \\
& =\sum_{z \in X} \underline{w(x, z)} \cdot \underline{w(z, x)} \\
& =\sum_{z \in X} \underline{w(x, z)} \cdot \underline{w(x, z)^{-1}} \\
& =\sum_{z \in X} \underline{\mathbf{1}}=n \underline{\mathbf{1}} .
\end{aligned}
$$

For $x \neq y$ we have:

$$
\begin{aligned}
\left(W^{2}\right)_{x, y} & =\sum_{z \in X}(W)_{x, z} \cdot(W)_{z, y} \\
& =\sum_{z \in X} w(x, z) \cdot w(z, y) \\
& =\sum_{z \in X} \delta w(y, x, z) \cdot w(x, y) \\
& =\left(\sum_{z \in X} \delta w(y, x, z)\right) \cdot \underline{w(x, y)} \\
& =\left((m(\mathbf{1})+2) \underline{\mathbf{1}}+m(\zeta) \underline{\zeta}+m\left(\zeta^{2}\right) \underline{\zeta^{2}}\right) \cdot \underline{w(x, y)} \\
& =\left((a+2) \underline{\mathbf{1}}+\underline{b, \zeta^{2}}\right) \cdot \underline{w(x, y)} .
\end{aligned}
$$

Summing up we get Equation (4.1).

Using:

$$
b \underline{\zeta, \zeta^{2}}(W-I)=b \underline{U_{3}} J-b \underline{U_{3}} I-b \underline{\mathbf{1}}(W-I)
$$

we get Equation (4.2).

Proposition 4.2. Every regular 3-graph with parameters $(n, a, b)$ defines a cyclic $(n, 3, b)$ cover.

Proof. We prove that the matrix $C=W-I$ is the Godsil-Hensel matrix of the cyclic cover $\Gamma_{w}$. We use Equation (4.2) to prove that $C$ satisfies the condition of Theorem 3.1.

$$
\begin{aligned}
C^{2}=(W-I)^{2} & =W^{2}-2 W+I \\
& =W^{2}-2(W-I)-I \\
& =n I+\left((a+2) \underline{\mathbf{1}}+b \underline{\zeta, \zeta^{2}}\right)(W-I)-2(W-I)-I \\
& =(n-1) I+\left(a \underline{\mathbf{1}}+b \underline{\zeta, \zeta^{2}}\right)(W-I) \\
& =(n-1) I+(a-b) \underline{\mathbf{1}}(W-I)+b \underline{U_{3}}(J-I) \\
& =(n-1) I+(a-b) \underline{\mathbf{1}} C+b \underline{U_{3}}(J-I) .
\end{aligned}
$$

Plugging in the values

$$
c_{2}=b, \quad a_{1}=a, \quad r=3
$$

we obtain Equation (3.1) in Theorem 3.1. 
The converse is proved similarly:

Proposition 4.3. Every cyclic $\left(n, 3, c_{2}\right)$-cover defines a regular 3-graph with parameters $\left(n, a_{1}, c_{2}\right)$.

Proof. Let $A$ be the Godsil-Hensel matrix of a cyclic $\left(n, 3, c_{2}\right)$-cover. We show that $W=$ $A+I$ is the matrix representation of a weight $w$ in the switching class of a regular 3-graph. We have:

$$
\begin{aligned}
W^{2}=(A+I)^{2} & =A^{2}+2 A+I \\
& =(n-1) I+\left(n-2-r c_{2}\right) A+c_{2} \underline{T}(J-I)+2 A+I \\
& =n I+\left(n-r c_{2}\right) A+c_{2} \underline{T}(J-I) \\
& =n I+\left(n-r c_{2}\right)(W-I)+c_{2} \underline{T}(J-I) .
\end{aligned}
$$

For the values

$$
b=c_{2}, \quad a=a_{1}, \quad r=3
$$

$W$ satisfies Equation (4.2) in Lemma 4.1.

To complete the picture we prove:

Proposition 4.4. There is a $1-1$ correspondence between regular 3-graphs and cyclic $\left(n, 3, c_{2}\right)$-covers.

Proof. Let $w$ and $w^{\prime}$ be weights into $U_{3}$. All that needs to be shown is:

$$
\delta w=\delta w^{\prime} \Longleftrightarrow \Gamma_{w} \cong \Gamma_{w^{\prime}}
$$

As was explained after Definition 2.10, the switching of a weight $w$ is interpreted as a cyclic permutation within the fibres of the corresponding cover $\Gamma_{w}$, thus switching equivalent weights yield isomorphic covers. The converse is straightforward.

As a consequence, using Theorem 9.2 of Godsil and Hensel in [2], we obtain a restriction on the parameter set of a regular 3-graph.

Corollary 4.5. If $(n, a, b)$ are the parameters of a regular 3 -graph then $3 \mid n$.

Proof. Since $\Gamma_{w}$ is a cyclic $(n, 3, b)$-cover, then by Theorem 9.2 in [2] we have $3 \mid n$.

\section{Higman's note: clarification and corrections}

\subsection{Regular 3-graphs and association schemes}

Higman's first step in [6] is to define $\Gamma_{w}=(V, E)$, an antipodal 3-fold cover of $K_{n}$, with fibre set $X$. He then constructs a rank 4 symmetric association scheme from $\Gamma_{w}$, this association scheme is (in a different ordering than the one that appears in [6]) the metric association scheme of the ADRG $\Gamma_{w}$. Higman's key observation is the fact that this rank 4 association scheme admits a rank 6 fission by orienting all the non-edges of $\Gamma_{w}$. We present this construction. 
Construction 5.1. Define:

$$
\begin{aligned}
& R_{0}=\operatorname{Id}_{V}, \\
& R_{1}=\left\{\left(x_{i}, x_{i+1} \quad(\bmod 3)\right) \mid i=1,2,3, x \in X\right\}, \\
& R_{2}=\left\{\left(x_{i}, x_{i+2} \quad(\bmod 3)\right) \mid i=1,2,3, x \in X\right\}, \\
& R_{3}=E, \\
& R_{4}=\left\{\left(x_{i}, y_{j}\right) \mid i=1,2,3,\left\{x_{i+1} \quad(\bmod 3), y_{j}\right\} \in E\right\}, \\
& R_{5}=\left\{\left(x_{i}, y_{j}\right) \mid i=1,2,3,\left\{x_{i+2} \quad(\bmod 3), y_{j}\right\} \in E\right\} .
\end{aligned}
$$

Remark 5.2. Notice that the relations $R_{1}, R_{2}, R_{4}, R_{5}$ are anti-symmetric, $R_{1}=R_{2}^{t}$ and $R_{4}=R_{5}^{t}$; Also $S_{1}=R_{1} \cup R_{2}$ is the "distance 3" relation and $S_{3}=R_{4} \cup R_{5}$ is the "distance 2 " relation with respect to $\Gamma_{w}=(V, E)$.

Proposition 5.3 (Higman). $\mathbb{A}_{6}(\Gamma):=\left(V,\left\{R_{i}\right\}_{i=0}^{5}\right)$ is an association scheme.

Proof. We calculate the intersection matrices of $\mathbb{A}_{6}(\Gamma)$. For example, we compute $p_{44}^{4}$ : let $\left(x_{i}, y_{j}\right) \in R_{4}$ and suppose $w\left(x_{i}, y_{j}\right)=\zeta$ (we may assume so due to switching), thus $j=i+1(\bmod 3)$. We count the number of $z_{k} \in V$ such that $\left(x_{i}, z_{k}\right) \in R_{4}$ and $\left(z_{k}, y_{j}\right) \in R_{4}$ : there are 3 types of $z \in X$ which contain such a $z_{k}$ :

• $k=i \Longrightarrow \begin{gathered}w(x, z)=\zeta^{2} \\ w(z, y)=\zeta\end{gathered} \Longrightarrow \delta w(x, y, z)=\zeta \cdot \zeta \cdot \zeta^{2}=\zeta$,

- $k=i+1(\bmod 3) \Longrightarrow \begin{aligned} & w(x, z)=\mathbf{1} \\ & w(z, y)=\mathbf{1}\end{aligned} \Longrightarrow \delta w(x, y, z)=\zeta \cdot \mathbf{1} \cdot \mathbf{1}=\zeta$,

- $k=i-1(\bmod 3) \Longrightarrow \begin{gathered}w(x, z)=\zeta \\ w(z, y)=\zeta^{2}\end{gathered} \Longrightarrow \delta w(x, y, z)=\zeta \cdot \zeta^{2} \cdot \zeta=\zeta$.

Thus $\delta w(x, y, z)=\zeta \Longleftrightarrow z$ is one of the above 3 types, hence $p_{44}^{4}=b$.

In the same manner we obtain the intersection matrices $\left\{B_{i}\right\}_{i=0}^{5}$ :

$$
\begin{aligned}
& B_{0}=\left[\begin{array}{llllll}
1 & 0 & 0 & 0 & 0 & 0 \\
0 & 1 & 0 & 0 & 0 & 0 \\
0 & 0 & 1 & 0 & 0 & 0 \\
0 & 0 & 0 & 1 & 0 & 0 \\
0 & 0 & 0 & 0 & 1 & 0 \\
0 & 0 & 0 & 0 & 0 & 1
\end{array}\right], B_{1}=\left[\begin{array}{llllll}
0 & 1 & 0 & 0 & 0 & 0 \\
0 & 0 & 1 & 0 & 0 & 0 \\
1 & 0 & 0 & 0 & 0 & 0 \\
0 & 0 & 0 & 0 & 1 & 0 \\
0 & 0 & 0 & 0 & 0 & 1 \\
0 & 0 & 0 & 1 & 0 & 0
\end{array}\right], B_{2}=\left[\begin{array}{llllll}
0 & 0 & 1 & 0 & 0 & 0 \\
1 & 0 & 0 & 0 & 0 & 0 \\
0 & 1 & 0 & 0 & 0 & 0 \\
0 & 0 & 0 & 0 & 0 & 1 \\
0 & 0 & 0 & 1 & 0 & 0 \\
0 & 0 & 0 & 0 & 1 & 0
\end{array}\right], \\
& B_{3}=\left[\begin{array}{cccccc}
0 & 0 & 0 & 1 & 0 & 0 \\
0 & 0 & 0 & 0 & 1 & 0 \\
0 & 0 & 0 & 0 & 0 & 1 \\
n-1 & 0 & 0 & a & b & b \\
0 & n-1 & 0 & b & a & b \\
0 & 0 & n-1 & b & b & a
\end{array}\right], B_{4}=\left[\begin{array}{cccccc}
0 & 0 & 0 & 0 & 1 & 0 \\
0 & 0 & 0 & 0 & 0 & 1 \\
0 & 0 & 0 & 1 & 0 & 0 \\
0 & n-1 & 0 & b & a & b \\
0 & 0 & n-1 & b & b & a \\
n-1 & 0 & 0 & a & b & b
\end{array}\right], \\
& B_{5}=\left[\begin{array}{cccccc}
0 & 0 & 0 & 0 & 0 & 1 \\
0 & 0 & 0 & 1 & 0 & 0 \\
0 & 0 & 0 & 0 & 1 & 0 \\
0 & 0 & n-1 & b & b & a \\
n-1 & 0 & 0 & a & b & b \\
0 & n-1 & 0 & b & a & b
\end{array}\right]
\end{aligned}
$$


It turns out that the existence of such a rank 6 association scheme is a sufficient condition:

Proposition 5.4 (Higman). Every rank 6 association scheme with parameters as in the construction of $\mathbb{A}_{6}\left(\Gamma_{w}\right)$ arises from a regular 3-graph.

Proof. Let $\left(V,\left\{R_{i}\right\}_{i=0}^{5}\right)$ be an association scheme with parameters (and notation) as in Construction 5.1. Define $T=R_{0} \cup R_{1} \cup R_{2}$, then $T$ is an equivalence relation on $V$ with equivalence classes of size 3. Denote $X=V / T$. We give a labeling of the elements of $V$ as a 3 -fold cover of $K_{|X|}$, and then we verify that in this cover we only have matchings of the 3 types shown in Figure 1. Let $a \in X$ be any fibre, and label its elements by $a_{1}, a_{2}, a_{3}$ so that $\left(a_{1}, a_{2}\right) \in R_{1}$. Then

$$
\left(a_{i}, a_{i+1}(\bmod 3)\right) \in R_{1}
$$

for $i=1,2,3$. We now label the elements of each fibre $x \neq a$ in $X$ by $x_{1}, x_{2}, x_{3}$ so that

$$
\left(a_{i}, x_{i}\right) \in R_{3}
$$

for $i=1,2,3$. To prove that $\left(V, R_{3}\right)$ is a 3 -fold cover of $K_{|X|}$ with matchings of the 3 permitted types, we prove two things:

(1) $\left(x_{i}, x_{i+1(\bmod 3)}\right) \in R_{1}$ for all $x \in X$ and $i=1,2,3$,

(2) there is no matching such that $\left(x_{i}, y_{j}\right) \in R_{3}$ and $\left(x_{j}, y_{i}\right) \in R_{3}$, where $i \neq j$.

Proof of (1): Assume that $\left(x_{i}, x_{i+1}\right) \in R_{2}$. Let $k$ be such that $\left(a_{i}, x_{i+1}\right) \in R_{k}$. Then we have:

$$
\left(x_{i}, a_{i}\right) \in R_{3}, \quad\left(x_{i}, x_{i+1}\right) \in R_{2}, \quad\left(x_{i+1}, a_{i}\right) \in R_{k^{\prime}} .
$$

Therefore:

$$
p_{2 k^{\prime}}^{3} \neq 0 \text {. }
$$

Examining column 3 in the matrix $B_{2}$, we deduce that $k^{\prime}=4$, which means that $k=5$. Also, we have:

$$
\left(a_{i}, x_{i+1}\right) \in R_{k}, \quad\left(a_{i}, a_{i+1}\right) \in R_{1}, \quad\left(a_{i+1}, x_{i+1}\right) \in R_{3} .
$$

This implies that:

$$
p_{13}^{k} \neq 0
$$

Examining row 3 in the matrix $B_{1}$, we deduce that $k=4$, which is a contradiction.

Proof of (2): Assume that $\left(x_{i}, y_{j}\right) \in R_{3}$ and $\left(x_{j}, y_{i}\right) \in R_{3}$ for some $i \neq j$. Let $k$ be such that $\left(x_{i}, y_{i}\right) \in R_{k}$. W.l.o.g we may assume that $j=i+1(\bmod 3)$. Then we have:

$$
\left(x_{i}, y_{i}\right) \in R_{k}, \quad\left(x_{i}, x_{i+1}\right) \in R_{1}, \quad\left(x_{i+1}, y_{i}\right) \in R_{3} .
$$

Therefore:

$$
p_{13}^{k} \neq 0 \text {. }
$$

Examining row 3 in the matrix $B_{1}$ we see $k=4$. Also, we have:

$$
\left(y_{i}, x_{i}\right) \in R_{k^{\prime}}, \quad\left(y_{i}, y_{i+1}\right) \in R_{1}, \quad\left(y_{i+1}, x_{i}\right) \in R_{3} .
$$


Thus we obtain:

$$
p_{13}^{k^{\prime}} \neq 0,
$$

which implies that $k^{\prime}=4$ and $k=5$, a contradiction.

It follows that all the matchings of the graph $\left(V, R_{3}\right)$ are of the 3 types shown in Figure 1 , and we can define a weight $w$ on $X$ by $w(x, x)=\mathbf{1}$ and $w(x, y)=\mathbf{1}, \zeta$ or $\zeta^{2}$ for $x \neq y$ according to as the matching is of the first, second or third type. It is straightforward to verify that $\delta w$ is regular.

\subsection{Characterization and feasibility conditions}

We now sum up the results of the previous sections with our characterization of regular 3graphs. Notice that the equivalence of $(i i)$ and $(i i i)$ is a characterization of cyclic $\left(n, 3, c_{2}\right)$ covers in terms of association schemes.

Corollary 5.5. Let $\Gamma$ be an antipodal 3-fold cover of $K_{n}$. The following are equivalent:

(i) $\Gamma$ defines a regular 3-graph with parameters $(n, a, b)$;

(ii) $\Gamma$ is a cyclic $(n, 3, b)$-cover;

(iii) $\mathbb{A}_{6}(\Gamma)$ is an association scheme.

Using this characterization we would like to obtain feasibility restrictions on the parameters $(n, a, b)$ of regular 3 -graphs. We begin by calculating the character-multiplicity tables of $\mathbb{A}_{4}\left(\Gamma_{w}\right)$ and $\mathbb{A}_{6}\left(\Gamma_{w}\right)$. We used the well-known computer software Mathematica to calculate these tables, the program code is presented in [7].

The character-multiplicity table of $\mathbb{A}_{4}\left(\Gamma_{w}\right)$ is:

$$
\left[\begin{array}{cccc}
1 & 2 & n-1 & 2(n-1) \\
1 & 2 & -1 & -2 \\
1 & -1 & \alpha & -\alpha \\
1 & -1 & \beta & -\beta
\end{array}\right]\left[\begin{array}{c}
1 \\
n-1 \\
z_{1} \\
z_{2}
\end{array}\right]
$$

Here:

- $\alpha$ and $\beta$ are the roots of $x^{2}-(a-b) x-(n-1)=0$,

- $z_{1}=\frac{2 n \beta}{\beta-\alpha}$,

- $z_{2}=2 n-z_{1}=\frac{2 n \alpha}{\alpha-\beta}$.

If $z_{1}=z_{2}=n$ then we have $\alpha=-\beta$, and $\alpha, \beta= \pm \sqrt{n-1}$.

Otherwise, $z_{2}-z_{1}$ is a non-zero integer, and we have:

$$
z_{2}-z_{1}=2 n\left(\frac{\alpha}{\alpha-\beta}-\frac{\beta}{\beta-\alpha}\right)=2 n\left(\frac{\alpha+\beta}{\alpha-\beta}\right) .
$$

This means that $\alpha-\beta=\sqrt{(a-b)^{2}+4(n-1)}$ is rational, i.e. $(a-b)^{2}+4(n-1)$ is a square, which implies that $\alpha$ and $\beta$ are rational algebraic integers, and thus are integers. 
The character-multiplicity table of $\mathbb{A}_{6}\left(\Gamma_{w}\right)$ is:

$$
\left[\begin{array}{cccccc}
1 & 1 & 1 & n-1 & n-1 & n-1 \\
1 & \zeta & \zeta^{2} & \alpha & \alpha \zeta & \alpha \zeta^{2} \\
1 & \zeta^{2} & \zeta & \alpha & \alpha \zeta^{2} & \alpha \zeta \\
1 & 1 & 1 & -1 & -1 & -1 \\
1 & \zeta & \zeta^{2} & \beta & \beta \zeta & \beta \zeta^{2} \\
1 & \zeta^{2} & \zeta & \beta & \beta \zeta^{2} & \beta \zeta
\end{array}\right]\left[\begin{array}{c}
1 \\
z_{1} / 2 \\
z_{1} / 2 \\
n-1 \\
z_{2} / 2 \\
z_{2} / 2
\end{array}\right]
$$

Here:

- $\alpha \zeta$ and $\alpha \zeta^{2}$ are the roots of $x^{2}+\alpha x+\alpha^{2}=0$,

- $\beta \zeta$ and $\beta \zeta^{2}$ are the roots of $x^{2}+\beta x+\beta^{2}=0$.

Remark 5.6. In Higman's note appeared the equations:

- $x^{2}-\alpha x+\left(\frac{3(n-1)}{2}+\alpha^{2}\right)=0$,

- $x^{2}-\beta x+\left(\frac{3(n-1)}{2}+\beta^{2}\right)=0$,

which led him to the false conclusion that $n$ must be odd. These equations are the result of a miscalculation of the intersection matrices $B_{3}$ and $B_{5}$ of $\mathbb{A}_{6}\left(\Gamma_{w}\right)$ (compare these matrices from our paper with those from the note [6]).

Summing up all the considered restrictions we obtain:

Proposition 5.7. Necessary conditions for the set $(n, a, b)$ of parameters of a regular 3graph are:

(i) $n=a+2 b+2$,

(ii) $3 \mid n$,

(iii) The roots $\alpha$ and $\beta$ of the equation $x^{2}-(a-b) x-(n-1)=0$ are integers,

(iv) $\alpha-\beta$ divides $n \alpha$.

Proof. Item $(i)$ appears in the beginning of Section 3. Item $(i i)$ is Corollary 4.5. Item (iii) comes from the latter analysis of the character-multiplicity table of $\mathbb{A}_{4}\left(\Gamma_{w}\right)$, and $(i v)$ is just the integrality of the multiplicity $\frac{z_{2}}{2}=\frac{n \alpha}{\alpha-\beta}$ of $\mathbb{A}_{6}\left(\Gamma_{w}\right)$.

These feasibility conditions provide a list of just 64 feasible parameter sets with $n \leq$ 1000. We refer to [7] for the complete list and details about known constructions for some of them.

\subsection{The symplectic example}

D. G. Higman provided an infinite family of regular 3-graphs which is described briefly below.

In [5], Higman considers a more general cohomological setting, and presents several group theoretic examples of regular 3-cocycles (here cochains are functions into a monoid with the appropriate conditions). These examples are mainly extensions of examples by 
D. E. Taylor in [15]. We mention one of them. In this example, we consider weights with values in the additive group of the field $G F(q)$, thus we will use additive notation: $C_{+}^{2}$ instead of $C_{.}^{2}, \delta_{+}$instead of $\delta$. etc.

Let $V$ be a $2 m$-dimensional vector space over $G F(q)$. Let $B$ be a non-degenerate alternating bilinear form on $V$. Then $B \in C_{+}^{2}(V, G F(q))$ is a weight on $V$ with values in $G F(q)$. In case $q$ is a prime, this 3-cocycle is a $q$-graph. To see that in this case $\Phi=\delta_{+} B$ is a regular $q$-graph we consider the symplectic group $S p(2 m, q)$. It acts transitively on the non-zero vectors of $V$, thus the subgroup $H:=V S p(2 m, q)$ of the affine group on $V$ acts 2-transitively on the vectors of $V$. The coboundary $\Phi=\delta_{+} B$ is invariant under translations and is therefore invariant under the action of $H$ on $V$. This provides an infinite family of regular $q$-graphs for every prime $q$.

\section{New constructions}

The equivalence of regular 3 -graphs with parameters $(n, a, b)$ and cyclic $(n, 3, b)$-covers provides a rich source of new examples of regular 3 -graphs.

In their recent paper [8], Klin and Pech present a construction of cyclic $\left(m^{2}, 3, \frac{m^{2}}{3}\right)$ covers from generalized Hadamard matrices of order $m$ over the cyclic group of order 3 ; the set of such matrices is denoted by $\mathrm{gH}\left(U_{3}, m\right)$. Their method takes as input any generalized Hadamard matrix $H \in \mathrm{gH}\left(U_{3}, m\right)$ and produces a so-called skew generalized Hadamard matrix $W \in \operatorname{gH}\left(U_{3}, m^{2}\right)$ of order $m^{2}$; such matrices correspond to cyclic $\left(m^{2}, 3, \frac{m^{2}}{3}\right)$ covers, this is the Godsil-Hensel matrix of the cover.

We used classifications of generalized Hadamard matrices with suitable parameters (see [3], [9] and [14]) to construct all the corresponding non-isomorphic cyclic covers using the Klin-Pech method, which provide different regular 3-graphs. A summary of our new constructions of regular 3-graphs:

- 1 new example with parameters $(36,10,12)$,

- 1 new example with parameters $(45,19,12)$ (exceptional),

- 1 new example with parameters $(81,25,27)$,

- 1 new example with parameters $(144,46,48)$,

- 28 new examples with parameters $(324,106,108)$.

For the complete list of feasible parameter sets with $n \leq 1000$, and details about the above examples see [7].

\section{Extension to regular $t$-graphs with $t \geq 4$}

The theory outlined in this paper can be extended to regular $t$-graphs with any $t \geq 4$ only if we impose certain restrictions on the parameters of the regular $t$-graph. For example, when 
$t$ is odd, the parameters of a regular $t$-graph are:

$$
\begin{aligned}
& n, \\
& m(1), \\
& m(\zeta)=m\left(\zeta^{t-1}\right), \\
& \vdots \\
& m\left(\zeta^{t-1 / 2}\right)=m\left(\zeta^{t+1 / 2}\right) .
\end{aligned}
$$

A graph $\Gamma_{w}$ defined by a regular $t$-graph will be distance regular only if most parameters of the regular $t$-graph are equal. Explicitly, in the case that $t$ is odd we demand:

$$
m(\zeta)=m\left(\zeta^{2}\right)=\cdots=m\left(\zeta^{t-1 / 2}\right)
$$

In this case, these will also be cyclic covers since for any $t$ we have

$$
C_{S_{t}}\left(C_{t}\right) \cong C_{t}
$$

Here we use the notation $C_{t} \leq S_{t}$ for the cyclic group $C_{t}=\langle(1,2, \ldots, t)\rangle$.

Higman's theory also extends to regular $t$-graphs with $t \geq 4$ in the case of equal parameters (as described above). The construction of $\mathbb{A}_{4}\left(\Gamma_{w}\right)$ is exactly the same, and it has a rank $2 t$ refinement which completely determines the weight $w$ (analogously to $\mathbb{A}_{6}\left(\Gamma_{w}\right)$ in the case of regular 3-graphs). Thus, the extension of our theory to $t \geq 4$ is described schematically in Figure 2:

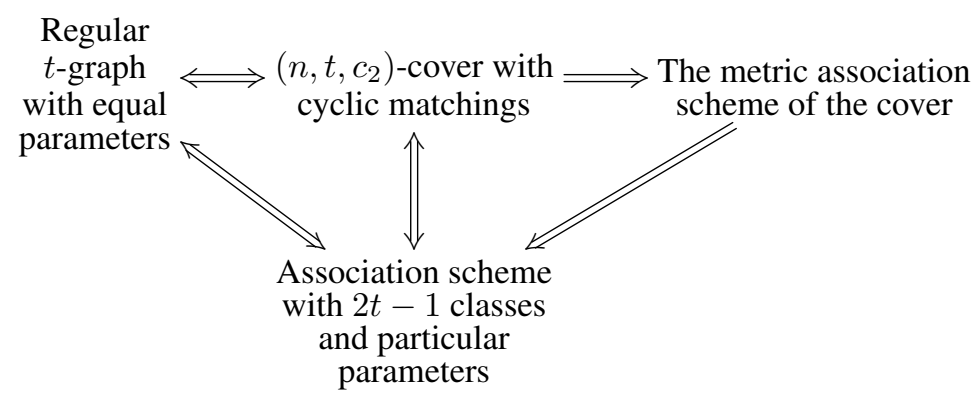

Figure 2: Extension to $t \geq 4$

\section{Acknowledgments}

The content of this paper is part of my MSc thesis completed in 2011 under the supervision of Misha Klin to whom I am thankful for his guiding hand. I thank Misha Muzychuk and Eran Nevo who were members of the committee for my thesis defence and gave helpful remarks. I thank Christian Pech whose valuable remarks on my thesis are reflected quite essentially in this paper. Finally, I thank the anonymous referees for their helpful remarks. 


\section{References}

[1] P. J. Cameron and J. H. van Lint, Graphs, codes and designs, volume 43 of London Mathematical Society Lecture Note Series, Cambridge University Press, Cambridge, 1980, revised edition of Graph theory, coding theory and block designs, an older version of Designs, Graphs, Codes and their Links.

[2] C. D. Godsil and A. D. Hensel, Distance regular covers of the complete graph, J. Combin. Theory Ser. B 56 (1992), 205-238.

[3] M. Harada, C. Lam, A. Munemasa and V. D. Tonchev, Classification of generalized Hadamard matrices $H(6,3)$ and quaternary Hermitian self-dual codes of length 18, Electron. J. Combin. 17 (2010), \#R171.

[4] D. G. Higman, Remark on Shult's graph extension theorem, in: Finite groups '72 (Proc. Gainesville Conf., Univ. Florida, Gainesville, Fla., 1972), North-Holland Math. Studies, Vol. 7, North-Holland, Amsterdam, 1973, pp. 80-83.

[5] D. G. Higman, Weights and t-graphs, Bull. Soc. Math. Belg. Sér. A 42 (1990), 501-521.

[6] D. G. Higman, A note on regular 3-graphs, 1994, unpublished draft.

[7] D. Kalmanovich, Regular t-graphs, antipodal distance regular graphs of diameter 3 and related combinatorial structures, MSc thesis, Ben-Gurion University of the Negev, 2011, http: //www. math. bgu.ac.il/ dannykal.

[8] M. Klin and C. Pech, A new construction of antipodal distance regular covers of complete graphs through the use of Godsil-Hensel matrices, Ars Math. Contemp. 4 (2011), 205-243.

[9] V. Mavron and V. Tonchev, On symmetric nets and generalized Hadamard matrices from affine designs, Journal of Geometry 67 (2000), 180-187.

[10] W. Mielants, A regular 5-graph, Atti Accad. Naz. Lincei Rend. Cl. Sci. Fis. Mat. Natur. (8), 60 (1976), 573-578.

[11] W. Mielants, Remark on the generalized graph extension theorem, European J. Combin. 1 (1980), 155-161.

[12] J. J. Seidel, A survey of two-graphs, in: Colloquio Internazionale sulle Teorie Combinatorie (Rome, 1973), Tomo I, pages 481-511, Atti dei Convegni Lincei, No. 17. Accad. Naz. Lincei, Rome, 1976.

[13] E. Shult, The graph extension theorem, Proceedings of the American Mathematical Society, 33 (1972), 278-284.

[14] C. Suetake, The classification of symmetric transversal designs $S T D_{4}[12 ; 3] \mathrm{s}$, Designs, Codes and Cryptography 37 (2005), 293-304.

[15] D. E. Taylor, Regular 2-graphs, Proc. London Math. Soc. 35 (1977), 257-274.

[16] D. E. Taylor and R. Levingston, Distance-regular graphs, in: Combinatorial mathematics (Proc. Internat. Conf. Combinatorial Theory, Australian Nat. Univ., Canberra, 1977), volume 686 of Lecture Notes in Math., Springer, Berlin, 1978, pp. 313-323. 